\title{
Mixed pathologies mimicking motor neuron disease: a case report and review of the literature
}

\author{
Avyarthana Dey ${ }^{1,2^{*}}$, Ekhlas Assaedi ${ }^{*}$, Wendy Johnston ${ }^{2}$, Sanjay Kalra ${ }^{1,2}$, Sumit Das ${ }^{1,3}$ \\ ${ }^{1}$ Neuroscience and Mental Health Institute, University of Alberta, Canada, ${ }^{2}$ Division of Neurology, University of Alberta, Canada, \\ ${ }^{3}$ Department of Laboratory Medicine and Pathology, University of Alberta, Canada \\ *Avyarthana Dey and Ekhlas Assaedi are co-first authors.
}

\begin{abstract}
Amyotrophic lateral sclerosis (ALS) is classified as a motor neuron disease (MND) that can present with both upper and lower motor neuron signs. Concurrent ALS and frontotemporal dementia (FTD) is also a well-known phenomenon. Examples of other primary disorders mimicking ALS or ALS-FTD have been reported in the literature and recognition of these entities is important to ensure proper clinical management. We present here an unusual case of an 86-year-old male patient, clinically diagnosed with ALS and severe cognitive impairment thought to be due to FTD. Postmortem neuropathological examination of his brain and spinal cord did not reveal the typical findings of ALS or FTD. Rather, it revealed multiple non-ALS pathologies including argyrophilic grain disease (AGD), cerebrovascular disease, and Alzheimer's type pathology. This case raises the possibility that mixed pathologies could mimic motor neuron disease.
\end{abstract}

Key words: amyotrophic lateral sclerosis, argyrophilic grain disease, cerebrovascular, Alzheimer's, neuropathology, overlap.

\section{Introduction}

Amyotrophic lateral sclerosis (ALS) is a neurodegenerative disorder that affects both upper motor neurons (UMNs) and lower motor neurons (LMNs). Along with progressive worsening motor functions, patients with ALS can also exhibit frontotemporal dementia (FTD)-like cognitive impairment [5]. Typically, the life expectancy of patients with ALS is 2-5 years, with death occurring as a result of respiratory insufficiency. While ALS is largely sporadic, familial cases are also possible. What seems to be underreported in the literature are the types of disorders that can mimic some of the clinical symptoms of ALS, thereby risking misdiagnosis and possibly inad- equate management. For example, Finsterer et al. reported an interesting case of a mitochondrial disorder presenting with slowly progressive weakness, wasting, and left upper limb fasciculations. Although the patient was diagnosed with ALS based on electrophysiological findings, muscle biopsy revealed histopathological findings in keeping with a mitochondrial disorder with combined complex II/III defect [6]. Consistent with ALS, atrophy of intrinsic hand muscle may also be seen in distal-type cervical spondylotic amyotrophy, Hirayama disease, and spinobulbar muscular atrophy [4]. The type of UMN degeneration typically associated with ALS may also be seen in primary lateral sclerosis. While examples of non-ALS pathology mimicking ALS seem 
to be sparse in the literature, it is important for neurologists and neuropathologists to be aware of this possibility to ensure timely clinical management.

The authors present here a patient who presented with weakness and cognitive decline and was diagnosed as "ALS with cognitive impairment" based on a combination of clinical and electrophysiological investigations. Post-mortem neuropathological examination however revealed findings unrelated to either ALS or the clinically defined cognitively impaired ALS (ALSCi).

\section{Case report}

An 86-year-old male was referred to our multidisciplinary amyotrophic lateral sclerosis clinic from an outside institution to evaluate decline in his strength and ambulation. The patient described a 6-month history of gradually progressing weakness of the upper and lower extremities with no sensory complaints. Other symptoms included word-finding difficulties and memory impairment with insidious onset. Past medical history was significant for hypertension, dyslipidemia, chronic obstructive pulmonary disease, coronary artery disease, and a remote left frontal subcortical stroke with near-complete recovery.

Montreal cognitive assessment (MOCA) scale revealed significant impairment in the recall, verbal fluency, and concentration, with a total score

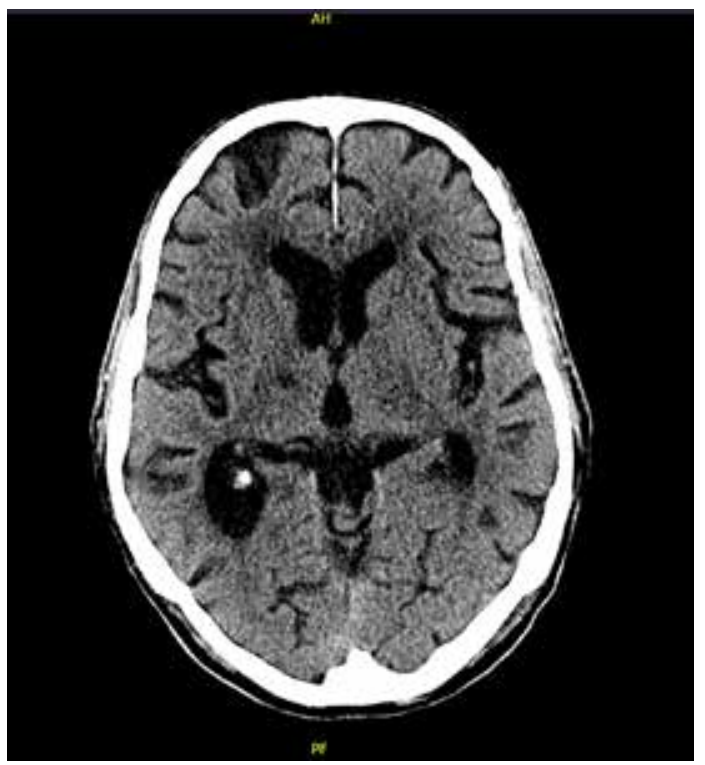

Fig. 1. Bilateral infarcts of the thalami and basal ganglia seen on unenhanced CT scan of the head. of $12 / 30$. Patient's cranial nerve examination was normal. Motor examination was notable for mild diffuse muscle atrophy. On power assessment, he had symmetric weakness of hip flexion (Medical Research Council grade [MRC] 3/5), ankle dorsiflexion (MRC grade 2/5), and bilateral hand weakness in first dorsal interossei and abductor pollicis brevis muscles (MRC grade 3/5). Hyperreflexia in the right upper extremity and a left Babinski sign were also present. Sensory nerve functions and coordination tests were normal. No fasciculations were observed. The patient walked with a stooped posture and a shuffling gait.

A computed tomography (CT) scan of the brain showed moderate diffuse cerebral atrophy, small vessel ischemic changes, and old perforator infarcts in the bilateral thalami and basal ganglia (Fig. 1). Magnetic resonance imaging (MRI) showed moderate to severe neural foraminal stenosis at the C3-4, C4-5, and C7-T1 levels of the spinal column. Moderate oropharyngeal dysphagia was evident in a video-fluoroscopic swallow study.

Sensory nerve conduction studies showed absent responses in the left median and bilateral ulnar nerves. Left superficial peroneal sensory study showed reduced amplitude and mildly reduced velocity. Sural nerve studies were not performed. Reduced amplitudes of compound muscle action potentials of the right peroneal nerve, bilateral tibial, and bilateral ulnar nerves were observed. Electromyography (EMG) showed acute on chronic denervation changes with fasciculations and reduced recruitment of bilateral tibialis anterior muscles, right quadriceps, right first dorsal interosseous, and right thoracic paraspinal. Chronic denervation changes were observed in the right deltoid. Left quadriceps showed chronic denervation changes with fasciculations. EMG of the right trapezius and the right genioglossus was normal. It was felt that the clinical presentation in conjunction with the electrodiagnostic studies, was suggestive of motor neuron disease (MND). Therefore, in accordance with the revised El Escorial criteria [2], a diagnosis of laboratory-supported probable ALS was provided.

Eight months after initial presentation, the patient developed progressive dysphagia, dysarthria, and mild sialorrhea. Four months later, he was transferred to a long-term care facility due to progressive cognitive and functional decline necessitating greater help with transfers and basic activities of daily living. Two years following the apparent symptom onset, a follow-up neurological assessment revealed 
a relatively stable examination without any further decline in cognitive, motor, or respiratory functions. With prolonged clinical stability and the relatively slow rate of weakness progression, the patient's course was felt to be atypical for ALS. At long-term care, the philosophy and goal of his care was deemed to be palliative. Thirty-six months following symptom onset, the patient passed away. Autopsy showed findings consistent with bronchopneumonia.

\section{Postmortem neuropathological findings}

Generalized muscle wasting was noted on post-mortem external examination of the patient. Mild-to-moderate cerebral atrophy was noted both before and after formalin-fixation of the brain. Post-fixation coronal sections of the cerebral hemispheres confirmed the cerebral atrophy, but no other significant abnormalities could be identified.
Microscopic examination of the cerebral hemispheres revealed neurofibrillary tangles, neurites, and neuropil threads throughout the orbitofrontal, temporal, cingulate, and calcarine cortices, demonstrated by tau immunohistochemistry. Acute-tosubacute microinfarcts were observed in the occipital cortex (Fig. 2A) and caudate nucleus. Chronic microinfarcts were observed in the pons (Fig. 2B) and thalamus (Fig. 2C, D). A subacute infarct was also observed in the pons that affected the crossing fibers. In the amygdala, neuritic plaques, tau-positive neurofibrillary tangles (Fig. 2E), pretangles, neurites, neuropil threads, argyrophilic grains, and balloon cells were observed (Fig. 2F). Subjacent white matter showed sparse tau-positive coiled bodies (Fig. 2G). The pretangles, argyrophilic grains, balloon cells and coiled bodies were felt to be in keeping with argyrophilic grain disease (AGD).
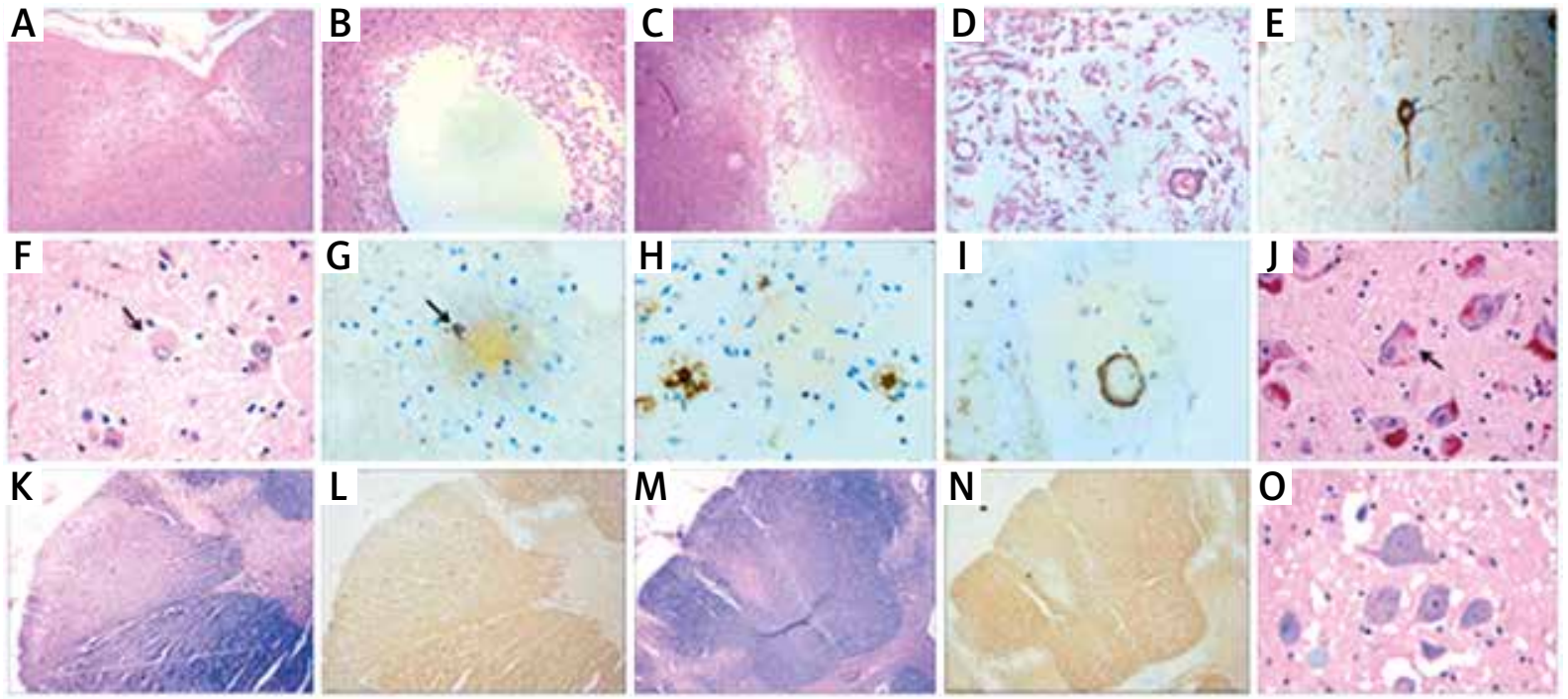

Fig. 2. A) Microinfarct in the occipital cortex observed as a slight discolouration in the gray matter and increased vacuolization as well as spongiosis changes. The image also shows thickened blood vessels in the leptomeninges. Magnification: 5x. B) Chronic infarct in the pons reveals well-defined infarct boundaries and a fluid-filled cystic space. Magnification: 10x. C) Acute infarct in the thalamus shows fragmented and no well-defined infarct boundary, identified as pale pink pallor of the tissue around the infarct location. Magnification: 10x. D) High magnification image of the thalamic infarct shows white matter axonal fragments and gliosis. Magnification: 20x. E) Tau-positive neurofibrillary tangle in the amygdala. F) Balloon cells (arrow) in the amygdala. G) Tau-positive coiled bodies (arrow). H) $\beta$-amyloid immunohistochemistry demonstrating neuritic plaques in the hippocampus. I) $\beta$-amyloid deposits around a parenchymal blood vessel in the amygdala. J) Lewy bodies (arrow) in the medulla. E-J) Magnification: 40x. K) Luxol fast blue stain demonstrating unilateral degeneration of the lateral corticospinal tract in the cervical spinal cord. L) Myelin basic protein stain demonstrating unilateral degeneration of the lateral corticospinal tract in the cervical spinal cord. M) Luxol fast blue stain demonstrating unilateral degeneration of the dorsal column in the cervical spinal cord. $\mathrm{N}$ ) Myelin basic protein stain demonstrating unilateral degeneration of the dorsal column in the cervical spinal cord. K-N) Magnification: 2.5×. O) Preserved anterior horn cells in the cervical spinal cord. Magnification: 40×. 
Some tau- and p62-positive neurofibrillary tangles, pre-tangles, neurites (Fig. $2 \mathrm{H}$ ), and neuropil threads were also found in the hippocampus, predominantly in the CA1 and subiculum subregions. Very few p62-positive but TDP-43-negative neuronal cytoplasmic inclusions were found in the dentate gyrus of the hippocampus. A relatively higher density of tau- and p62-positive neurofibrillary tangles, neurites, and neuropil threads were observed in the adjacent entorhinal cortex. Scattered $\beta$-amyloid-positive diffuse plaques and a relatively moderate amount of neuritic plaques were also observed in the entorhinal cortex. In addition, some leptomeningeal and parenchymal blood vessels showed $\beta$-amyloid positive amyloid deposits in the vessel walls (Fig. 2l).

Rare non-specific p62-positive and TDP-43-negative neuronal cytoplasmic inclusions were identified in the motor nucleus of Vagus in the medulla. Alpha-synuclein-positive Lewy bodies were also identified in the medulla (Fig. 2J). The hypoglossal nucleus did not show any apparent neuronal loss, which was thought to be atypical for ALS.

Microscopically, sections of cervical, thoracic, and lumbosacral spinal cord showed unilateral degeneration of the lateral corticospinal tract and dorsal column with Luxol fast blue staining and myelin basic protein immunohistochemistry, suggestive of Wallerian degeneration. This was most appreciated at the level of the cervical spinal cord (Fig. 2K-N). No significant loss of motor neurons (MNs) was observed in the anterior horn of the spinal cord or the precentral gyrus (PCG, Fig. 20). The population of Betz cells in the PCG appeared spared. No obvious neuronal or glial inclusions were observed with TDP-43 immunohistochemistry.

The final neuropathological diagnoses were: 1) tau pathology consistent with AGD; 2) cerebral amyloid angiopathy (CAA); 3) Alzheimer's type neuropathological changes [amyloid deposition score of A1 (Thal phase 2); Braak staging (neurofibrillary tangle) of III/IV; CERAD scoring (neuritic plaque) of C2 or moderate]; 4) recent and old infarcts (occipital cortex, basal ganglia, thalamus, and pons).

\section{Discussion}

This case demonstrates the ability of mixed nonALS pathologies to mimic the clinical phenotype of ALS with cognitive impairment. Accumulation of phosphorylated TDP-43 in neurons and glia is a key neuropathological finding in ALS but this was not observed in our patient. Instead, post-mortem examination of our patient's brain revealed evidence of concomitant neurodegenerative conditions and cerebrovascular findings. The most prominent neurodegenerative pathology found in our patient were those in keeping with AGD. AGD has been reported both as a distinct entity and to occur concurrently with other neurodegenerative disorders including progressive supranuclear palsy $[7,19,20]$, corticobasal degeneration $[7,19,20]$, multiple system atrophy [19], vascular dementia [7], and Alzheimer's disease (AD) $[1,7,19]$. This observation, along with its age-dependency and existence in non-demented subjects, might have contributed to the longstanding controversy of its existence as a distinct disease entity [1,11]. Multiple authors have reported AGD presenting clinically as FTD and this was suspected in our patient $[8,10]$.

Liang et al. reported a 61-year-old female patient whose clinical phenotype was felt to be in keeping with ALS-parkinsonism. She progressed to quadriparesis and died from cardiopulmonary arrest. Postmortem neuropathological examination revealed findings correlating with the history of parkinsonism in the form of depigmentation of the substantia nigra and locus coeruleus along with alpha-synuclein positive Lewy bodies in the brainstem, striatum, amygdala, and cingulate gyrus. Additional findings included loss of motor neurons in the anterior horn of the spinal cord, brainstem and motor cortex, myelin loss in lateral and anterior corticospinal tracts, and ubiquitin positive (non-reactive to tau, $\alpha$-synuclein, peripherin, $\alpha B$-crystallin, $\alpha$-internexin antibodies) eosinophilic inclusions in the remaining motor neurons. Tau-positive argyrophilic grains were found in the hippocampus, entorhinal cortex and amygdala [14]. This case demonstrates potential complexities in clinical presentation and in turn the difficulties of clinical diagnosis that can arise in cases with multiple neuropathological processes as was the case with our patient. Hedera et al. reviewed another patient with a history of dementia and examination findings of extrapyramidal signs and MND. Pathologically, mixed findings consistent with AD, Lewy body dementia (LBD), Parkinson's disease (PD), and ALS were found [9]. Clinicopathologic studies investigating dementia highlight the high prevalence of mixed pathologies. In one study that included 50 patients with dementia who underwent autopsy, half received multiple neuro- 
pathological diagnoses. AD pathology with infarcts was the most common mixed pathology in this study followed by AD with PD/LBD [16]. In a larger cohort, which included 447 patients with probable $A D, 47 \%$ of patients were found to have AD, vascular, and mixed pathologies [16]. The literature seems to suggest that patients with mixed pathologies have a lower threshold for dementia than those with a single pathological diagnosis [12,15].

Our patient's cognitive impairment was of late-onset, which would be consistent with the increasing prevalence of AGD with age [11]. Moreover, one study noticed a higher proportion of cerebrovascular pathology, as in our patient, in AGD patients [7]. Cerebrovascular disease can present with symptoms secondary to cortical and/or subcortical damage which include aphasia, apraxia, seizures, abnormal gait, incontinence, parkinsonism, and executive dysfunction. Cognitive changes can vary in severity from vascular cognitive impairment to vascular dementia [13]. Microinfarcts were reported in subjects with vascular dementia, mixed dementia, as well as non-demented subjects [17]. The risk of dementia is likely increased by both microinfarcts and lacunar infarcts. It has been suggested that the threshold for manifestation of dementia, in the presence of moderate AD pathology, is lowered when cortical microinfarcts or lacunar infarcts are present $[3,18]$. It is therefore plausible that the cerebrovascular findings in our patient may have contributed to the presentation of cognitive symptoms.

To the authors' knowledge, the literature regarding cerebrovascular pathology mimicking ALS or other motor neuron diseases is scarce. The neuropathological observations in our patient possibly correlating motor symptoms noted clinically may be somewhat unique. It could be argued that cerebrovascular pathology was a sufficient explanation for the patient's weakness and dementia, and the coexistence of AGD pathology, CAA, microinfarct in the thalamus, and Alzheimer's-type neuropathological changes, could have contributed to a lower threshold for dementia.

The authors acknowledge the lack of genetic testing and sampling of skeletal muscle which might have provided a more complete post-mortem examination. In addition, serial brain imaging was not available to compare the relevance of the infarcts to the temporal course of his disease. The patient's EMG findings remain unexplained.
Herein the authors have presented a case of mixed neuropathological findings from multiple neurodegenerative conditions that mimicked the ALSci clinical phenotype. More research is needed to determine the precise relationships between concomitant pathologies and manifestation of MND phenotypes with or without cognitive decline. This case report also highlights the pitfalls of making a clinical and neurophysiological diagnosis of ALS in elderly patients, particularly those with atypical course of progression.

\section{Acknowledgements}

We thank the family for consenting to the use of this case for educational purposes.

\section{Disclosure}

The authors report no conflict of interest.

\section{References}

1. Braak H, Braak E. Argyrophilic grain disease: Frequency of occurrence in different age categories and neuropathological diagnostic criteria. J Neural Transm (Vienna) 1998; 105: 801-819.

2. Brooks BR, Miller RG, Swash M, Munsat TL. El Escorial revisited: Revised criteria for the diagnosis of amyotrophic lateral sclerosis. Amyotroph Lateral Scler Other Motor Neuron Disord 2000; 1: 293-299.

3. Esiri MM, Nagy Z, Smith MZ, Barnetson L, Smith AD. Cerebrovascular disease and threshold for dementia in the early stages of Alzheimer's disease. Lancet 1999; 354: 919-920.

4. Fang J, Liu MS, Guan YZ, Du H, Li BH, Cui B, Ding QY, Cui LY. Pattern differences of small hand muscle atrophy in amyotrophic lateral sclerosis and mimic disorders', Chinese Medical Journal (Engl) 2016; 129: 792-798.

5. Ferrari R, Kapogiannis D, Huey ED, Momeni P. FTD and ALS: a tale of two diseases. Curr Alzheimer Res 2011; 8: 273-294.

6 . Finsterer J. Consequences of misdiagnosing mitochondrial disorder. Int J Neurosci 2006; 116: 907-914.

7. Gil MJ, Sagrario Manzano M, Cuadrado ML, Fernández C, Góméz E, Matesanz C, Calero M, Rábano A. Argyrophilic grain pathology in frontotemporal lobar degeneration: demographic, clinical, neuropathological, and genetic features. J Alzheimers Dis 2018; 63: 1109-1117.

8. Gil MJ, Serrano S, Sagrario Manzano M, Cuadrado ML, Góméz E, Rábano A. Argyrophilic grain disease presenting as behavioral frontotemporal dementia. Clin Neuropathol 2019; 38: 8-13.

9. Hedera P, Lerner AJ, Castellani R, Friedland RP. Concurrence of Alzheimer's disease, Parkinson's disease, diffuse Lewy body disease, and amyotrophic lateral sclerosis. J Neurol Sci 1995; 128: 219-224.

10. Ishihara K, Araki S, Ihori N, Shiota J, Kawamura M, Yoshida M, Hashizume Y, Nakano I. Argyrophilic grain disease presenting with frontotemporal dementia: A neuropsychological and 
pathological study of an autopsied case with presenile onset. Neuropathology 2005; 25: 165-170.

11. Josephs KA, Whitwell JL, Parisi JE, Knopman DS, Boeve BF, Geda YE, Jack CR Jr, Petersen RC, Dickson DW. Argyrophilic grains: A distinct disease or an additive pathology? Neurobiol Aging 2008, 29: 566-573.

12. Kapasi A, DeCarli C, Schneider JA. Impact of multiple pathologies on the threshold for clinically overt dementia. Acta Neuropathol 2017; 134: 171-186.

13. Korczyn AD, Vakhapova V, Grinberg LT. Vascular dementia. J Neurol Sci 2012; 322: 2-10.

14. Liang TW, Forman MS, Duda JE, McCluskey L, Trojanowski JQ Siderowf A. Multiple pathologies in a patient with a progressive neurodegenerative syndrome. J Neurol Neurosurg Psychiatry 2005; 76: 252-255.

15. Schneider JA, Arvanitakis Z, Bang W, Bennett DA. Mixed brain pathologies account for most dementia cases in community-dwelling older persons. Neurology 2007; 69: 2197-2204.

16. Schneider JA, Aggarwal NT, Barnes L, Boyle P, Bennett DA. The neuropathology of older persons with and without dementia from community versus clinic cohorts. J Alzheimers Dis 2009; 18: 691-701.

17. Smith EE, Schneider JA, Wardlaw JM, Greenberg SM. Cerebral microinfarcts: The invisible lesions. Lancet Neurol 2012; 11: 272 282.

18. Snowdon DA, Greiner LH, Mortimer JA, Riley KP, Greiner PA, Markesbery WR. Brain infarction and the clinical expression of Alzheimer disease. The Nun Study. JAMA 1997; 277: 813-817.

19. Soma K, Fu YJ, Wakabayashi K, Onodera O, Kakita A, Takahashi H. Co-occurrence of argyrophilic grain disease in sporadic amyotrophic lateral sclerosis. Neuropathol Appl Neurobiol 2012; 38: 54-60.

20. Togo T, Sahara N, Yen SH, Cookson N, Ishizawa T, Hutton M, de Silva R, Lees A, Dickson DW. Argyrophilic grain disease is a sporadic 4-repeat tauopathy. J Neuropathol Exp Neurol 2002; 61: 547-556. 